Artículo Original

\title{
Calculo de la generación de biogás para el relleno sanitario de la ciudad de Juliaca, utilizando el modelo LandGEM Versión 3.02 de la USEPA y estimación del potencial de producción eléctrica
CALCULATION OF GENERATION OF BIOGAS FOR THE SANITARY OF THE USEPA AND ESTIMATION OF THE POTENTIAL OF ELECTRIC PRODUCTION FILLING OF JULIACA CITY, USING THE LandGEM MODEL VERSION 3.02
}

\author{
GLEN BRYAN MADRIGAL PÉREZ§, JUAN JOSE QUISPE SAAVEDRA§, YOEL MODESTO \\ VARGAS HUAMAN§*
}

Recibido: 15 septiembre de 2018 / Aceptado: 07 noviembre de 2018

$\S E P$. Ingeniería Ambiental, Facultad de Ingeniería y Arquitectura, Universidad Peruana Unión, Perú.

\begin{abstract}
Resumen
Los vertederos y rellenos sanitarios constituyen una fuente importante de biogás resultado de la descomposición del proceso biológico de residuos sólidos orgánicos, conformado principalmente por $\mathrm{CH}_{4} \mathrm{y}$ $\mathrm{CO}_{2}$. Existen modelos matemáticos que permiten estimar la producción de biogás en la fuente final de los residuos sólidos municipales (RSM). Por ello, el objetivo de esta investigacion fue estimar la produccion del biogas en fuente del relleno sanitario que se construirá para la ciudad de Juliaca consiederando los residuos sólidos orgánicos que se encuentran en él, para ello se aplicó el modelo "Landfill Gas Emissions Model" (LandGEM, version 3.02). Según nuestros resultados se podrían generar 1.226.945.217 $\mathrm{m}^{3}$ de biogás con una media de 29.925.493,10 $\mathrm{m}^{3}$ de biogás/año llegando a su producción máxima en el año 2044 con 58.285.801,66 $\mathrm{m}^{3}$ biogás/año. En cuanto a la energía total de cogeneración, se estima que podrían llegar a una capacidad máxima de 284.113 MWh/año en el año 2044, además de que la media de producción es de $145.871 \mathrm{MWh} / \mathrm{año}$ de los cuales $82.721 \mathrm{MWh} / \mathrm{año}$ corresponden a energía térmica y 63.151 MWh/año a energía eléctrica.

Palabras claves: metano, energía, vertedero, relleno sanitario, residuos
\end{abstract}

\begin{abstract}
Landfills and landfills are an important source of biogas resulting from the decomposition of the biological process of organic solid waste, consisting mainly of $\mathrm{CH}_{4}$ and $\mathrm{CO}_{2}$. There are mathematical models that allow estimating the production of biogas in the final source of municipal solid waste (MSW). Therefore, the objective of this research was to estimate the production of biogas at the source of the sanitary landfill that will be built for the Juliaca city with organic solid waste that is in it, for it was applied the model "Landfill Gas Emissions Model" (LandGEM, version 3.02). According to our results, 1,226,945,217 $\mathrm{m}^{3}$ of biogas could be generated with an average of $29,925,493.10 \mathrm{~m}^{3}$ of biogas/year, reaching its maximum production in 2044 with 58,285,801.66 m3 biogas / year. In terms of total cogeneration energy, it is estimated that they could reach a maximum capacity of $284,113 \mathrm{MWh} /$ year in the year 2044 , in addition to the average production of $145,871 \mathrm{MWh}$ /year of which $82,721 \mathrm{MWh} /$ year correspond to thermal energy and 63,151 MWh / year to electric power.

Keyword: methane, energy, landfill, landfill, waste
\end{abstract}




\section{INTRODUCCIÓN}

La gran necesidad actual de preservar y mantener el medio ambiente y la urgencia de ser cada día menos dependientes de los combustibles fósiles, nos dirige hacia el objetivo de buscar nuevas alternativas energéticas que sean menos contaminantes el cual sean explotadas produciendo un impacto ambiental mínimo (Pérez, 2006). Este cambio a nuevas fuentes energéticas que sean renovables y sostenibles es motivado por la problemática del cambio climático y principalmente del calentamiento global ocasionado por el uso indiscriminado de los combustibles fósiles (Quintero y Quintero, 2015). Al utilizar combustibles fósiles ocurre un incremento considerable de gases de invernadero (ver tabla 1) y algunos pronósticos establecen que para el año 2100, la temperatura sufrirá un incremento que va de $1.5^{\circ} \mathrm{C}$, en un escenario conservador, hasta $4.5^{\circ} \mathrm{C}$ en el escenario extremo (Aguirre, Camacho y Solorzano).

Los residuos sólidos municipales experimentan un crecimiento progresivo como consecuencia del aumento poblacional dado a que existe una relación directa entre la generación y caracterización de residuos con la cantidad de habitantes (Jaramillo y Zapata, 2008) producen cantidades significativas de metano $\left(\mathrm{CH}_{4}\right)$, dióxido de carbono biogénico $\left(\mathrm{CO}_{2}\right)$ y compuestos orgánicos volátiles diferentes del metano (COVDM), así como también cantidades pequeñas de óxido nitroso $\left(\mathrm{N}_{2} \mathrm{O}\right)$, óxidos de nitrógeno y monóxido de carbono (CO) (IPCC, 2006). El conjunto de todos estos compuestos es el llamado "biogás", el metano es de suma importancia por sus efectos en la regulación térmica de la tierra, pues posee un poder 21 veces mayor de retención de radiación solar comparado con el dióxido de carbono (Armenta-rivas, Sierra-Camargo y Vélez-Pereira, 2017; Batool y Chuadhry, 2008) y que el metano en los sitios de eliminación de desechos sólidos contribuye aproximadamente un 3 a un 4 por ciento de las emisiones de gases de efecto invernadero antropogénicas mundiales anuales (IPCC, 2006).

En definitiva, el biogás es una mezcla de metano de 40 a $70 \%$, dióxido de carbono y otros gases (hidrogeno, sulfuros, etc.) generado por la acción de microorganismos especializados para descomponer la materia orgánica dentro del relleno sanitario. La cantidad de biogás producido en los lugares de disposición final, es función de la cantidad de residuos el tipo y edad, contenido de humedad, la temperatura y las prácticas de manejo (Martínez y Rodríguez, 2016).

La estimación de las emisiones de biogás en los sitios de disposición final ha sido analizada por diversos investigadores, principalmente para conocer su potencial como fuente renovable de energía y mitigación de emisiones de gases de efecto invernadero (Aguilar, Ojeda, Taboada y Quintero, 2012). Como resultado de estas investigaciones se han generado diversos modelos para estimar y/o predecir la generación de biogás, tales como; el modelo de la EPA modelo de degradación de primer orden, modelo LandGEM, método IPCC y el modelo mexicano de Biogás.

Es ampliamente reconocido que el biogás es una fuente potencialmente significativa de energía (Aguilar, Taboada y Ojeda, 2011), así mismo, muchas veces se encuentran en las plantas que generan energía eléctrica a partir del biogás es una buena solución ya que es muy eficiente, similar a la de las plantas térmicas a gas que se utilizan en la actualidad (Panesso, Cadena, Mora y Ordoñez, 2011). 
Por tanto, en nuestro se estima la generación de biogás del relleno sanitario proyectado para la ciudad de Juliaca-San Roman - Puno, para ello se utilizará el modelo matemático "Landfill Gas Emissions Model" (LandGEM, versión 3.02). Además, se calcula la producción de energía eléctrica teniendo encuentra su potencial.

En un relleno sanitario o en su defecto en un botadero, el agua y los residuos son principalmente los insumos, mientras que el lixiviado y el gas son el producto de la descomposición de todos los desechos (Machado, Carvalho, Gourc, Vilar y Nascimento, 2009). El biogás producido en un relleno sanitario está constituido por un conjunto de gases resultado de la descomposición de la fracción orgánica de los residuos sólidos urbanos en condiciones anaeróbicas (fermentación en ausencia de oxigeno) (Pérez, 2006; Aguilar, Taboada, y Ojeda, 2011). Si este gas no se aprovecha puede ser una fuente muy importante de generación de gases de efecto invernadero y en consecuencia uno de los principales responsables del cambio climático.

El metano, por su alto contenido energético puede ser capturado y utilizado para la generación de energía eléctrica o combustible para el transporte. El uso de este recurso para generar calor y electricidad es cada vez más usado en los países desarrollados (Córdoba, Santalla, y Blanco, 2009), especialmente en el continente Europeo. El que se hayan desarrollado varias aplicaciones en el área de la combustión, calefacción, iluminación y motores que accionan generadores eléctricos es debido a que el biogás tiene un poder calorífico aproximado de 4.700 a $5.500 \mathrm{kcal} / \mathrm{m}^{3}$ (Valladares y Villegas, 2013).

\section{MATERIALES Y MÉTODOS}

\section{LUGAR DE ESTUDIO}

La investigación se realizó en la ciudad de Juliaca ubicada en la provincia de San Román en el departamento de Puno, en sureste de Perú. Esta ciudad se encuentra a una altitud de 3824 m.s.n.m. ocupa parte de la meseta altiplánica de Toropampa, en la cuenca del rio Coata, sección Ayabaca, desarrollándose entre los cerros Zapatiana, de la Cruz y Huaynaroque. Se encuentra asimismo atravesada de este a oeste por el rio Torococha, que desemboca en el rio Coata y continua su curso hasta desembocar en el lago Titicaca. Juliaca cuenta con una población de 225.146 habitantes (2007).

Actualmente el botadero de Chilla se ubica en las coordenadas mostradas en la tabla $1 \mathrm{y}$ próximamente se construirá un relleno sanitario en el mismo lugar, en este sentido la estimación de la generación de biogás será proyectado para el tiempo de vida del relleno sanitario que son 25 años, que se prevé que empezará a funcionar en el 2018, en caso de que no empiece a funcionar el relleno sanitario no afectan los cálculos, simplemente se desplazan los años, de acuerdo a la fecha de operación del relleno sanitario (siempre tener en cuenta que los datos hallados en el presente artículo son referenciales).

La estimación de biogás generado se llevó a cabo siguiendo dos puntos: se realizó una investigación sobre las cantidades y características de los residuos y se siguieron los lineamientos propuestos en el modelo matematico "Landfill Gas Emissions Model" (LandGEM, version 3.02). 
Tabla 1. Coordenadas de ubicación del botadero Chilla

\begin{tabular}{llc}
\hline Puntos & \multicolumn{2}{c}{ Coordenadas UTM “Zona 19" } \\
\cline { 2 - 3 } & Este X & Norte Y \\
\hline Punto 1 & 381566 & 1713688 \\
Punto 2 & 381563 & 1713959 \\
Punto 3 & 381860 & 1713970 \\
Punto 4 & 381865 & 1713806 \\
\hline
\end{tabular}

\section{CONSIDERACIONES TÉCNICAS}

\section{Cantidad y composición de residuos en el relleno}

La información de las cantidades, composición de los residuos sólidos generados en la ciudad de Juliaca provienen del informe presentado por la municipalidad provincial de San Román en su PIGARS. El estudio de caracterización de los residuos sólidos se realizó en el año 2015. En este sentido, la generación per-cápita total de los residuos sólidos municipales en el distrito de Juliaca (Área urbana) es de: 0,72 kg/hab/dií y 198,26 t/día (Tabla 2).

Tabla 2. Generación per-cápita y cantidad de residuos sólidos municipales en Juliaca

\begin{tabular}{cccccc}
\hline tipo & Generación & Población & $\begin{array}{c}\text { Generación total } \\
\mathrm{kg} / \mathrm{dia}\end{array}$ & $\begin{array}{c}\text { Generación total } \\
\text { t/día }\end{array}$ & $\begin{array}{c}\text { Generación total } \\
\text { t/año }\end{array}$ \\
\hline $\begin{array}{c}\text { GPC de RS } \\
\text { municipales } \\
\text { (kg/hab/dia) }\end{array}$ & 0,72 & 275.361 & $198.259,92$ & 198,26 & $71.897,79$ \\
\hline
\end{tabular}

Estimación de la generación de residuos sólidos municipales. En tal sentido a continuación se presenta la proyección de la generación total de residuos sólidos municipales al 2043 como fecha estipulada con el fin de alimentar el modelo.

Tabla 1. Proyección de la generación de residuos sólidos municipales en la ciudad de Juliaca

\begin{tabular}{|c|c|c|c|c|c|c|}
\hline Generació & ta de $\mathrm{R}$ & & & $=$ & 0.72 & $\mathrm{~kg} / \mathrm{hab} / \mathrm{dia}$ \\
\hline Tasa de cr & poblac & & & $=$ & 3.04 & $\%$ \\
\hline tasa de cre & de RSN & & & $=$ & 3.04 & $\%$ \\
\hline población & o de es & 015) & & $=$ & 275361 & hab. \\
\hline Nro & AÑO & Población & $\begin{array}{c}\text { GPC } \\
\mathrm{kg} / \mathrm{hab} / \mathrm{día}\end{array}$ & $\begin{array}{l}\text { Generación de } \\
\text { RSM Ton/día }\end{array}$ & $\begin{array}{c}\text { Generación } \\
\text { RSM Ton/mes }\end{array}$ & $\begin{array}{c}\text { Generación RSM } \\
\text { Ton/año }\end{array}$ \\
\hline 0 & 2015 & 275.361 & 0,72 & 198,26 & $5.947,80$ & $72.364,87$ \\
\hline 1 & 2016 & 283.732 & 0,742 & 210,50 & $6.314,92$ & $76.831,53$ \\
\hline 2 & 2017 & 292.357 & 0,764 & 223,49 & $6.704,70$ & $8.1573,89$ \\
\hline 3 & 2018 & 301.245 & 0,788 & 237,28 & $7.118,55$ & $86.608,97$ \\
\hline 4 & 2019 & 310.403 & 0,812 & 251,93 & $7.557,93$ & $91.954,84$ \\
\hline 5 & 2020 & 319.839 & 0,836 & 267,48 & $8.024,44$ & $97.630,67$ \\
\hline 6 & 2021 & 329.562 & 0,862 & 283,99 & $8.519,74$ & $103.656,85$ \\
\hline 7 & 2022 & 339.581 & 0,888 & 301,52 & $9.045,61$ & $110.054,98$ \\
\hline 8 & 2023 & 349.904 & 0,915 & 320,13 & $9.603,95$ & $116.848,03$ \\
\hline 9 & 2024 & 360.541 & 0,943 & 339,89 & $10.196,74$ & $124.060,38$ \\
\hline 10 & 2025 & 371.502 & 0,971 & 360,87 & $10.826,13$ & $131.717,90$ \\
\hline 11 & 2026 & 382.795 & 1,001 & 383,15 & $11.494,36$ & $139.848,07$ \\
\hline 12 & 2027 & 394.432 & 1,031 & 406,79 & $12.203,84$ & $148.480,08$ \\
\hline 13 & 2028 & 406.423 & 1,063 & 431,90 & $12.957,11$ & $157.644,89$ \\
\hline
\end{tabular}




\begin{tabular}{ccccccc}
14 & 2029 & 418.778 & 1,095 & 458,56 & $13.756,88$ & $167.375,39$ \\
\hline \multicolumn{1}{c}{ Continuación de tabla } & & & & & \\
\hline 15 & 2030 & 431.509 & 1,128 & 486,87 & $14.606,01$ & $177.706,49$ \\
16 & 2031 & 444.627 & 1,163 & 516,92 & $15.507,56$ & $188.675,27$ \\
17 & 2032 & 458.144 & 1,198 & 548,82 & $16.464,75$ & $200.321,10$ \\
18 & 2033 & 472.071 & 1,234 & 582,70 & $17.481,02$ & $212.685,75$ \\
19 & 2034 & 486.422 & 1,272 & 618,67 & $18.560,02$ & $225.813,60$ \\
20 & 2035 & 501.21 & 1,311 & 656,85 & $19.705,62$ & $239.751,75$ \\
21 & 2036 & 516.446 & 1,350 & 697,40 & $20.921,94$ & $254.550,23$ \\
22 & 2037 & 532.146 & 1,391 & 740,44 & $22.213,33$ & $270.262,13$ \\
23 & 2038 & 548.324 & 1,434 & 786,15 & $23.584,42$ & $286.943,83$ \\
24 & 2039 & 564.993 & 1,477 & 834,67 & $25.040,15$ & $304.655,20$ \\
25 & 2040 & 582.168 & 1,522 & 886,19 & $26.585,74$ & $323.459,78$ \\
26 & 2041 & 599.866 & 1,569 & 940,89 & $28.226,72$ & $343.425,07$ \\
27 & 2042 & 618.102 & 1,616 & 998,97 & $29.968,99$ & $364.622,69$ \\
28 & 2043 & 636.893 & 1,665 & 1060,63 & $31.818,80$ & $387.128,72$ \\
\hline
\end{tabular}

También es importante el conocer la composición de los residuos sólidos municipales que en el caso de la ciudad de Juliaca (Figura 1).

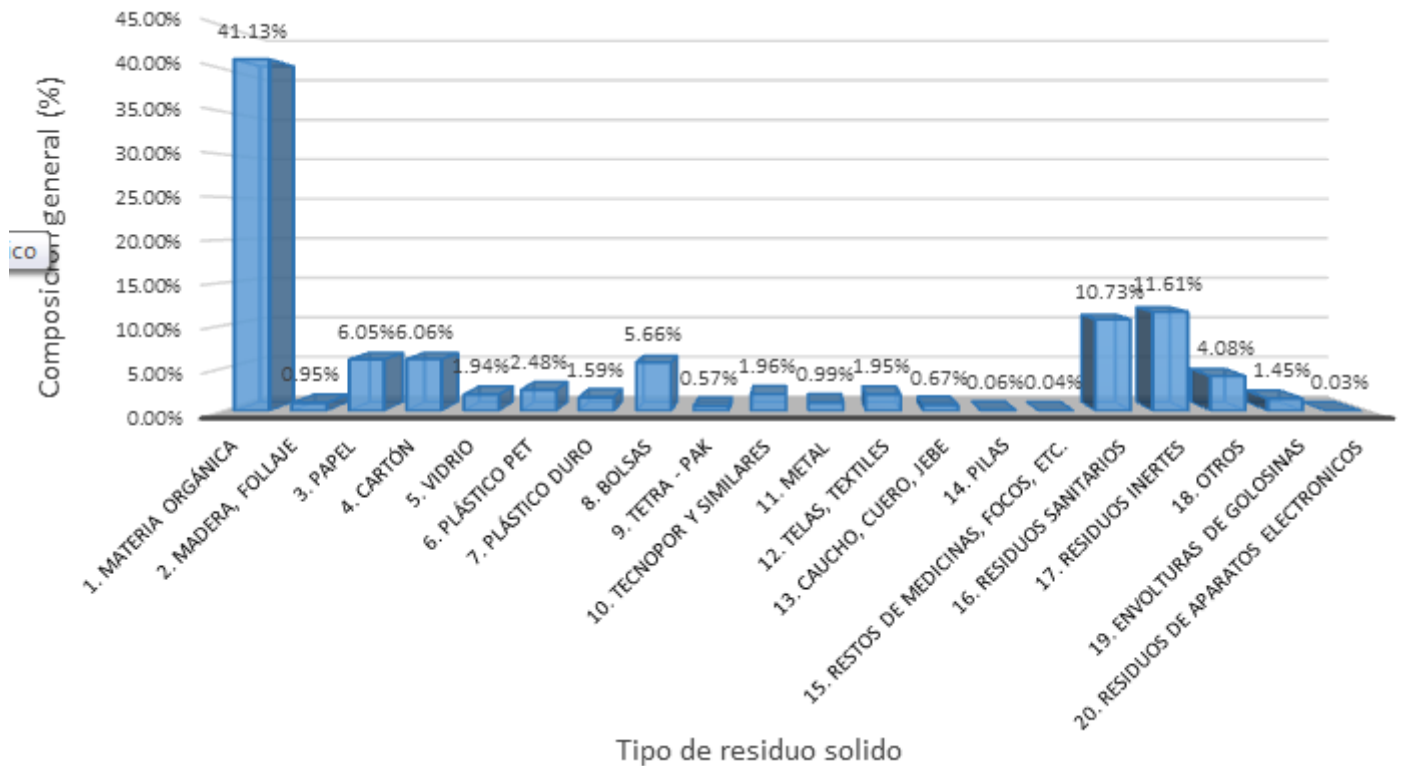

Figura 1. Composición de Residuos Sólidos Municipales generados en la ciudad de Juliaca

Como se puede ver en la figura precedente, la composicion de materia orgánica es del 41 $\%$ y si consideramos la composición de madera, follaje, papel y cartón (que también son considerados materiales orgánicos) el porcentaje total de material orgánico es de $54.19 \%$ un poco mas de la mitad del total de residuos sólidos que se produce en la ciudad de Juliaca. Esta composición de materia orgánica es común en muchas partes del mundo.

Estimación de la generación de biogás por el modelo matematico "Landfill Gas Emissions Model" (LandGEM, version 3.02).

La estimación del biogás emitido desde sitios de disposicion final de residuos sólidos es uno de los objetivos previstos en el protocolo de Kioto; de hecho, resulta fundamental evaluar la contribucion de los rellenos sanitarios, botaderos y plantas de compostaje a lo largo del tiempo por la producción de biogás, así como establecer medidas que puedan 
adoptarse para la reduccion de los gases emitidos (Pignato, Aronica, Bonanno, Piazza, \& Trapani, 2008)

Para la cuantificación del biogás se aplicará el modelo matemático "Landfill Gas Emissions Model" (landGEM) versión 3.02. las particularidades de aplicación para el modelo se describen a continuación.

\section{Modelo landGEM version 3.02}

El modelo Landfill Gas Emission Model (LandGEM) de la Agencia de Protección Ambiental de Estados Unidos (EPA), se utiliza para estimar las emisiones de sitios de disposicion de residuos sólidos municipales, como también es una herramienta de estimación automatizada, encuadrada en el programa de cálculo "Microsoft Excel". Las emisiones estimadas producidas en una vertedero son tanto el biogás, como de cada uno de los gases componentes del mismo como el metano, el dioxido de carbono, los componentes orgánicos que no sean metano y otros tipos de contaminantes atmosféricos que se podrían producir en el mismo.

LandGEM es uno de los modelos que se basa en una ecuación de descomposicion con cinética de primer orden para estimar la generación de biogás con el tiempo. La ecuación se expresa así:

Donde,

$$
Q_{C H_{4}}=\sum_{t=1}^{n} \sum_{j=0.1}^{1} k L_{0}\left(\frac{M_{i}}{10}\right) e^{-k t_{i j}}
$$

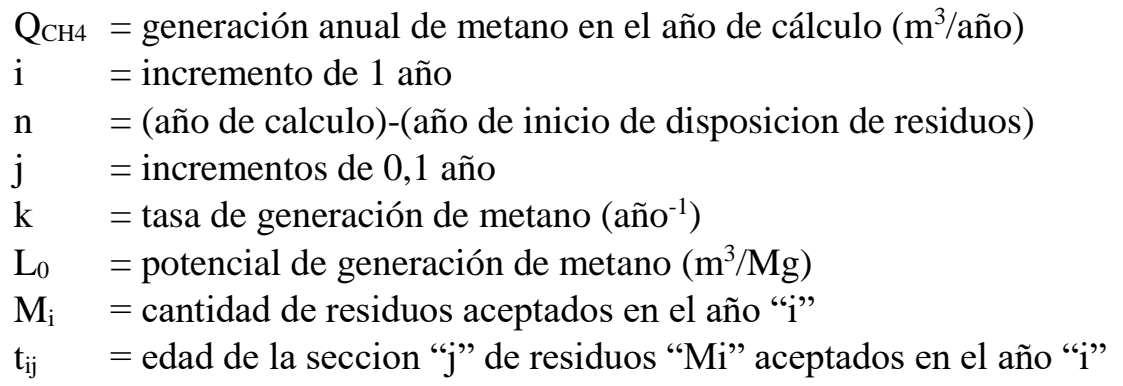

El modelo utiliza dos tipos de factores de emisión por defecto para la $\mathrm{k}$ (constante de generación de biogás) y la $\mathrm{L}_{0}$ (ptencial de generación de biogás). La primera esta influida por la temperatura, humedad, disponibilidad de nutrientes y el ph y la segunda por el tipo y la composición de los RSM. Los valores por defecto son los de la CAA (Clean Air ACT) basados en normativa de EEUU y los de inventario.

Los valores de la constante de degradación y el potencial de generación de metano se han elegido según los valores por defecto que proporciona el modelo, los valores de $\mathrm{k}_{\mathrm{y}} \mathrm{L}_{0} \mathrm{se}$ pueden observar en la tabla 4.

Tabla 2. Parámetros para k y Lo para rellenos convencionales, modelo EPA

\begin{tabular}{cc}
\hline Parámetros del modelo & Valor \\
\hline $\mathrm{k}$ & 0,050 por año \\
$\mathrm{L}_{0}$ & $170 \mathrm{~m}^{3} /$ ton \\
\hline
\end{tabular}

Fuente: EPA. Landfill Gas Emissions Model. 2006 
Los datos que requiere el LandGEM para estimar las emisiones que se van a producir en un vertedero de RSM, son básicamente el año de apertura, el de clausura y la cantidad anual de RSM depositados, así como los parámetros $\mathrm{k}$ y L $\mathrm{L}_{0}$ por defecto o calculándolas con los datos característicos de cada instalación y los gases contaminantes que se desee estimar.

\section{Estimación del potencial de producción eléctrica}

Para estimar la producción eléctrica se considerará la utilización de una planta de cogeneración, esto debido a que la utilización de la cogeneración en el sector ambientalista se ha situado en los vertederos y en las estaciones depuradoras de aguas residuales (Pérez, 2006) en función a la experiencia acumulada en diferentes paises. Para evaluar la energía cogenerada a partir del biogás se tomará en consideracion el motor de combustión interna (Chamy \& Vivanco, 2007), ya que representa las mejores condiciones para las necesidades energéticas que nos interesan, tanto en potencia, tamaño e inversión.

Un parámetro caracteristico importante en las centrales eléctricas de cogeneración, en nuestro caso particular de biogás, es el rendimiento, tanto el rendimiento de energía térmica y de energía eléctrica, así como también los rendimientos de los equipos de recuperación de calor de los equipos de transferencia de calor que captan la energía disipada del motor en forma de calor (Chamy \& Vivanco, 2007).

El biogás puede ser utilizado como una fuente potencial de energía debido a su alto poder calorífico, para efectos de calculo, el valor del poder calorífico sera el que menciona Camargo y Vélez (2009), Eurostat y la Agencia Internacional de la Energía (AIE) como 20 $\mathrm{MJ} / \mathrm{m}^{3}$. Es así como el biogás puede utilizarse de forma directa como energía térmica o generando energía eléctrica.

En resumen, para determinar la producción de energía por medio de una planta de cogeneración usando biogás se requiere el poder calorífico del biogás, el rendimiento de energía eléctrica y de energía térmica, así como también los rendimientos de los equipos de recuperación de calor de los equipos de transferecia de calor.

\section{RESULTADOS Y DISCUSIÓN}

En los siguientes apartados se muestran los resultados de las proyecciónes de generación de biogás en el relleno sanitario proyectado así como también el potencial de producción eléctrica en una planta de cogeneración. En cuanto a la proyección (como se menciono con anterioridad) se utilizo el "Landfill Gas Emissions Model" (LandGEM) que es una herramienta de estimación automatizada, encuadrada en el programa de cálculo Microsoft Excel”.

Es muy importante considerar que en este estudio, para estimar el potencial energético se supondrá un mínimo de 15 años después del cierre del relleno sanitario, tiempo esperado para concentrar un alto volumen de biogás y la estabilizacion del $\mathrm{CH}_{4}$. Esto quiere decir que para realizar los cálculos de biogás y especialmente del potencial eléctrico producido se tomaran datos desde el año 2018 al año 2058. 


\section{Estimación del biogás producido}

Para calcular el potencial de producción de biogás en el relleno sanitario de Juliaca, se utilizó el modelo LandGEM, con los siguientes datos: Cantidad de RSM depositados (ver tabla 4), año de apertura del relleno: 2018, año de clausura: 2043, contenido de metano $50 \%, \mathrm{k}=0,05 \mathrm{año}^{-1} \mathrm{y} \mathrm{L}_{0}=170 \mathrm{~m}_{\mathrm{CH}}^{3} /$ megagramos.

En base a los supuestos citados y a la cantidad de RSM depositados, se logró determinar el volumen generado de biogás, $\mathrm{CH}_{4}, \mathrm{CO}_{2}$ y NMOC. Los valores respectivos se encuentran tabulados a continuación.

Tabla 3. Volumen generado de biogás, $\mathrm{CH}_{4}, \mathrm{CO}_{2}$ y NMOC

\begin{tabular}{|c|c|c|c|c|}
\hline Año & $\begin{array}{l}\text { Total Landfill } \\
\text { gas (m³/año }\end{array}$ & 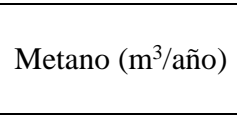 & $\begin{array}{c}\text { Dióxido de } \\
\text { carbóno } \\
\text { (m³/año) }\end{array}$ & $\begin{array}{c}\text { NMOC } \\
\left(\mathbf{m}^{\mathbf{3}} / \mathbf{a} \tilde{\mathbf{n} o}\right)\end{array}$ \\
\hline 2018 & 0,00 & 0,00 & 0,00 & 0,00 \\
\hline 2019 & $1.439 .742,99$ & $719.871,49$ & $719.871,49$ & $5.758,97$ \\
\hline 2020 & $2.898 .135,81$ & $1.449 .067,91$ & $1.449 .067,91$ & $11.592,54$ \\
\hline 2021 & $4.379 .754,14$ & $2.189 .877,07$ & $2.189 .877,07$ & $17.519,02$ \\
\hline 2022 & $5.889 .289,06$ & $2.944 .644,53$ & $2.944 .644,53$ & $23.557,16$ \\
\hline 2023 & $7.431 .562,34$ & $3.715 .781,17$ & $3.715 .781,17$ & $29.726,25$ \\
\hline 2024 & $9.011 .542,25$ & $4.505 .771,12$ & $4.505 .771,12$ & $36.046,17$ \\
\hline 2025 & $10.634 .359,96$ & $5.317 .179,98$ & $5.317 .179,98$ & $42.537,44$ \\
\hline 2026 & $12.305 .326,64$ & $6.152 .663,32$ & $6.152 .663,32$ & $49.221,31$ \\
\hline 2027 & $14.029 .951,17$ & $7.014 .975,59$ & 7.014.975,59 & $56.119,80$ \\
\hline 2028 & $15.813 .958,79$ & $7.906 .979,39$ & 7.906.979,39 & $63.255,84$ \\
\hline 2029 & $17.663 .310,37$ & $8.831 .655,19$ & $8.831 .655,19$ & $70.653,24$ \\
\hline 2030 & $19.584 .222,81$ & $9.792 .111,41$ & $9.792 .111,41$ & $78.336,89$ \\
\hline 2031 & $21.583 .190,22$ & $10.791 .595,11$ & $10.791 .595,11$ & $86.332,76$ \\
\hline 2032 & $23.667 .006,25$ & $11.833 .503,13$ & $11.833 .503,13$ & $94.668,03$ \\
\hline 2033 & $25.842 .787,54$ & $12.921 .393,77$ & $12.921 .393,77$ & $103.371,15$ \\
\hline 2034 & $28.117 .998,33$ & $14.058 .999,17$ & $14.058 .999,17$ & $112.471,99$ \\
\hline 2035 & $30.500 .476,38$ & $15.250 .238,19$ & $15.250 .238,19$ & $122.001,91$ \\
\hline 2036 & 32.99 & 16.4 & $16.499 .230,16$ & $131.993,84$ \\
\hline 2037 & 35.620 .6 & 0.309 & $17.810 .309,19$ & $142.482,47$ \\
\hline 2038 & $38.376 .078,81$ & $19.188 .039,40$ & $19.188 .039,40$ & $153.504,32$ \\
\hline 2039 & $41.274 .461,88$ & $20.637 .230,94$ & $20.637 .230,94$ & $165.097,85$ \\
\hline 2040 & $44.325 .913,79$ & $22.162 .956,90$ & $22.162 .956,90$ & $177.303,66$ \\
\hline 2041 & $47.541 .142,40$ & $23.770 .571,20$ & $23.770 .571,20$ & $190.164,57$ \\
\hline 2042 & $50.931 .455,05$ & $25.465 .727,52$ & $25.465 .727,52$ & $203.725,8202$ \\
\hline 2043 & $54.508 .798,58$ & $27.254 .399,29$ & $27.254 .399,29$ & $218.035,1943$ \\
\hline 2044 & $58.285 .801,66$ & $29.142 .900,83$ & $29.142 .900,83$ & $233.143,2066$ \\
\hline 2045 & 55443169,57 & $27.721 .584,78$ & $27.721 .584,78$ & $221.772,6783$ \\
\hline 2046 & 52739174,28 & $26.369 .587,14$ & $26.369 .587,14$ & $210.956,6971$ \\
\hline 2047 & 50167054,4 & $25.083 .527,2$ & $25.083 .527,2$ & $200.668,2176$ \\
\hline 2048 & 47720378,28 & $23.860 .189,14$ & $23.860 .189,14$ & $190.881,5131$ \\
\hline 2049 & 45393027,97 & $22.696 .513,99$ & $22.696 .513,99$ & $181.572,1119$ \\
\hline 2050 & 43179183,88 & $21.589 .591,94$ & $21.589 .591,94$ & $172.716,7355$ \\
\hline 2051 & 41073310,23 & $20.536 .655,11$ & $20.536 .655,11$ & $164.293,2409$ \\
\hline 2052 & 39070141,25 & $19.535 .070,63$ & $19.535 .070,63$ & $156.280,565$ \\
\hline 2053 & 37164667,98 & $18.582 .333,99$ & $18.582 .333,99$ & $148.658,6719$ \\
\hline 2054 & 35352125,73 & $17.676 .062,87$ & $17.676 .062,87$ & $141.408,5029$ \\
\hline 2055 & 33627982,21 & $16.813 .991,11$ & $16.813 .991,11$ & $134.511,9289$ \\
\hline 2056 & 31987926,17 & $15.993 .963,08$ & $15.993 .963,08$ & $127.951,7047$ \\
\hline 2057 & 30427856,6 & $15.213 .928,3$ & $15.213 .928,3$ & $121.711,4264$ \\
\hline 2058 & 28943872,52 & $14.471 .936,26$ & $14.471 .936,26$ & $115.775,4901$ \\
\hline TOTAL & 1226945217,00 & $613.472 .608,50$ & $613.472 .608,50$ & $4.907 .780,87$ \\
\hline
\end{tabular}


En la Figura 2 se muestra las curvas de generación de biogás, $\mathrm{CH}_{4}, \mathrm{CO}_{2}$ y $\mathrm{NMOC}$ en metros cúbicos por año y megagramos por año.

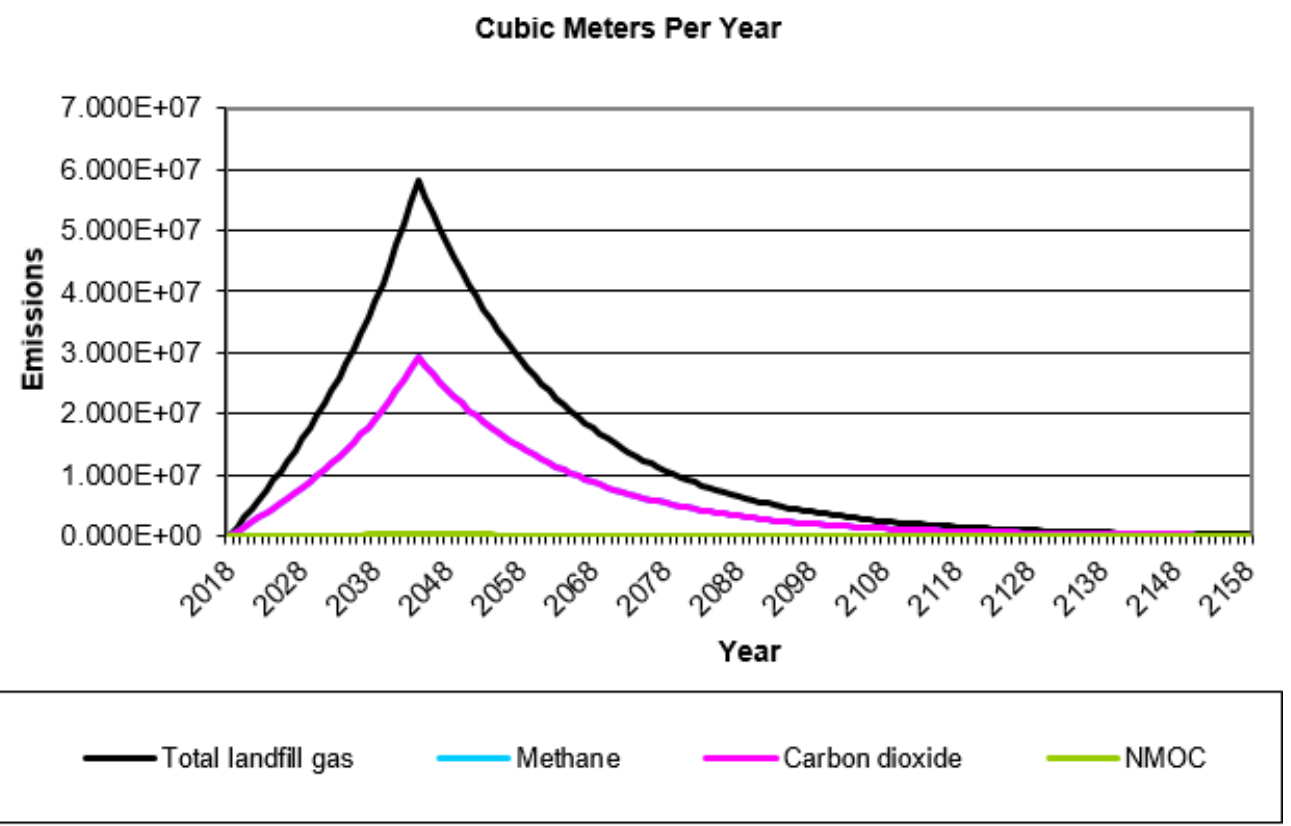

Figura 1.Variacion de biogás estimado por el modelo en $\mathrm{m}^{3} /$ año

Megagrams Per Year

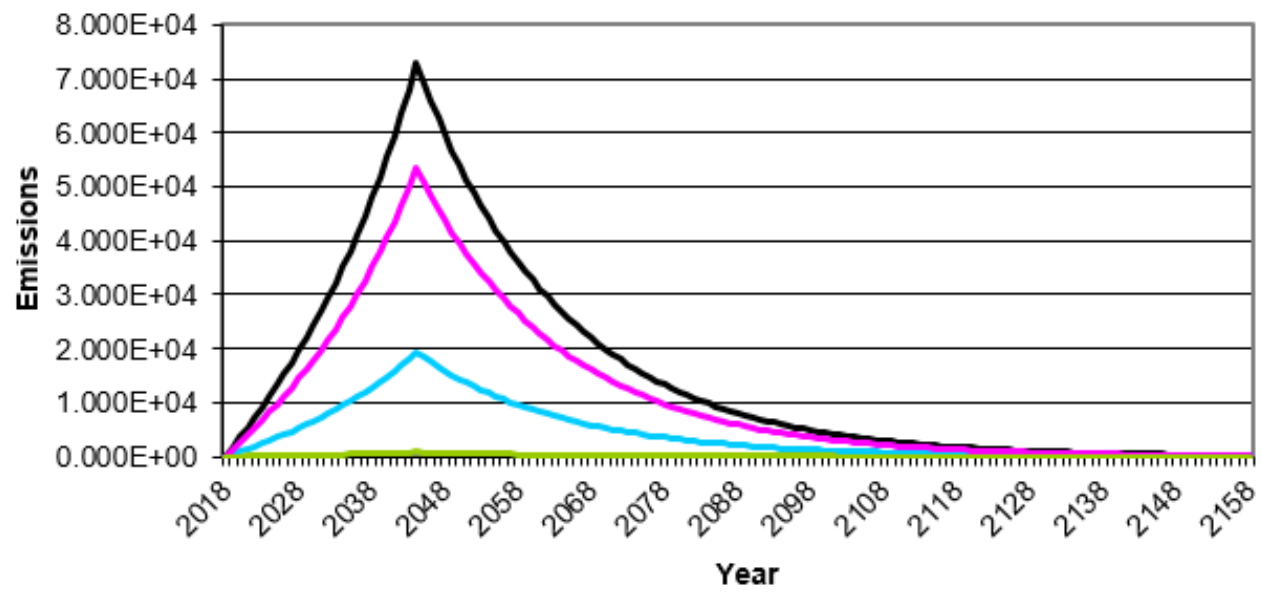

Total landfill gas

Methane $\quad$ Carbon dioxide

NMOC

Figura 2. Variación de biogás estimado por el modelo en Mg/año

Los resultados que arroja el programa comprende el periodo de 2018 hasta el año 2158, periodo en el cual se producirá biogás. Es de aclarar que los datos de estudio planteado asumen un inicio en el año 2018, con una productividad proyectada hasta el año 2043. 
Según este modelo, y considerando una composición media del biogás de $50 \%$ de $\mathrm{CH}_{4} \mathrm{y}$ $50 \%$ de $\mathrm{CO}_{2}$, y que comience la captura de biogás en el 2018 según los valores de $\mathrm{k}$ y $\mathrm{L}_{0}$ descritos el potencial de recuperación de biogás estimado llegara a su punto más alto un año después del año de clausura, es decir, en el año 2044 con 58.285.801,66 mªño. Posteriormente disminuirá, año tras año. Esto se presenta porque el modelo asume que la generación máxima normalmente ocurre en el año de clausura o al año siguiente, y la generación de biogás desciende exponencialmente en la medida en que la fracción orgánica de los residuos es consumida (SCS Engineers, 2009).

El promedio de biogás generado para los años de 2018 a 2058 corresponde a 29.925.493,1 $\mathrm{m}^{3} /$ año, es una cantidad importante considerando el aprovechamiento energético que se le puede dar.

\section{Cálculo de la energía cogenerada a partir de biogás}

Se calculará la energía generada para los años 2018 al 2058. Para efectos de cálculo se considera la cantidad anual de biogás generado a partir de los RSM y un poder calorífico de $20 \mathrm{MJ} / \mathrm{m}^{3}=4775 \mathrm{kcal} / \mathrm{m}^{3}$ de biogás $(1000 \mathrm{kcal}=1,163 \mathrm{kWh})$.

A continuación, para ilustrar el procedimiento seguido se mostrará como ejemplo la secuencia de cálculo utilizada para calcular la energía generada a partir de los RSM, como dato se utilizará la producción pico o máxima de biogás que es de 58.285.801,66 mªño.

Energía térmica recuperada de los gases del motor de combustión

De los 58.285.801,66 m³/año. de biogás, la energía disponible disipada en los gases de combustión es de un $31.8 \%$ de la energía aportada por el combustible, la que a su vez pasara por la caldera de recuperación que tiene un rendimiento del $65 \%$ entonces:

$$
\begin{gathered}
E_{\text {disponible }}=58.285 .801,66 \frac{\mathrm{m}^{3} \text { biogás }}{a \tilde{n} o} \cdot 4.775 \frac{\mathrm{kcal}}{\mathrm{m}^{3}} \cdot 0,318 \cdot 0,65=5,75 \cdot 10^{10} \frac{\mathrm{kcal}}{a \tilde{\mathrm{n} o}} \\
5,75 \cdot 10^{10} \frac{\mathrm{kcal}}{a \tilde{\mathrm{n} o}}=66.873 \frac{\mathrm{MWh}}{a \tilde{n} o} \\
E_{\text {disponible }}=66.905 \frac{\mathrm{MWh}}{a \tilde{\mathrm{n} o}}
\end{gathered}
$$

Agua de enfriamiento

De los 58.285.801,66 m³/año. de biogás, la energía disponible en el agua de alta temperatura $\left(90\right.$ a $\left.99^{\circ} \mathrm{C}\right)$ es de un $19,7 \%$ que pasara por un intercambiador de calor de un rendimiento del $98 \%$, entonces:

$$
\begin{gathered}
E_{\text {disponible }}=58.285 .801,66 \frac{\mathrm{m}^{3} \text { biogás }}{a \tilde{n} o} \cdot 4.775 \frac{\mathrm{kcal}}{\mathrm{m}^{3}} \cdot 0,197 \cdot 0,98=62.490 \frac{\mathrm{MWh}}{a \tilde{\mathrm{n} o}} \\
E_{\text {disponible }}=62.490 \frac{\mathrm{MWh}}{a \tilde{\mathrm{n} o}}
\end{gathered}
$$

De los 58.285.801,66 m³/año de biogás, la energía disponible en el agua de baja temperatura, inferior a $50^{\circ} \mathrm{C}$ es de un $10 \%$ que también pasara por un intercambiador de 
calor de rendimiento del $98 \%$ entonces:

$$
\begin{gathered}
E_{\text {disponible }}=58.285 .801,66 \frac{\mathrm{m}^{3} \text { biogás }}{a \tilde{n} o} \cdot 4.775 \frac{\mathrm{kcal}}{\mathrm{m}^{3}} \cdot 0,1 \cdot 0,98=31.721 \frac{\mathrm{MWh}}{a \tilde{n} o} \\
E_{\text {disponible }}=31.721 \frac{\mathrm{MWh}}{a \tilde{n} o}
\end{gathered}
$$

Esta energía de baja temperatura se intentará utilizarla para calentar los reactores de producción de biogás que deben estar a unos $35^{\circ} \mathrm{C}$. la energía térmica total cogenerada a partir de los sistemas de enfriamiento, será la suma de ambos sistemas tanto de baja como de alta temperatura.

$$
E_{\text {disponible }}=62.490+31.721=94.211 \frac{M W h}{a \tilde{n} o}
$$

La energía térmica total cogenerada será la suma de la energía de gases y la energía de agua de enfriamiento de alta temperatura.

$$
E_{\text {disponible }}=66.905+62.490+31.721=161.115 \frac{M W h}{a \tilde{n} o}
$$

\section{Energía eléctrica recuperada a partir del biogás}

La energía eléctrica recuperada del biogás corresponde a un 38\% de esta, por tanto:

$$
\begin{gathered}
E_{\text {disponible }}=58.285 .801,66 \frac{\mathrm{m}^{3} \text { biogas }}{a \tilde{\mathrm{n} o}} \cdot 4.775 \frac{\mathrm{kcal}}{\mathrm{m}^{3}} \cdot 0,38=122.998 \frac{M W h}{a \tilde{\mathrm{n} o}} \\
E_{\text {disponible }}=122.998 \frac{\mathrm{MWh}}{a \tilde{\mathrm{n} o}}
\end{gathered}
$$

De acuerdo a los valores mostrados, la energía generada a partir de biogás en el año de mayor producción (2044) en este caso es de 122.998 MWh/año. Finalmente, la energía total cogenerada a partir de biogás correspondiente a los residuos sólidos municipales es de 284.113 MWh/año (122.998 MWh/año como energía eléctrica y 161.115 MWh/año como energía térmica).

En la siguiente Tabla 6 y Figura 4 se detallan la energía cogenerada a partir de biogás en MWh/año, según los $\mathrm{m}^{3}$ de biogás calculados. 
Tabla 4. Cantidad de energía producida por el proceso de cogeneración

\begin{tabular}{|c|c|c|c|c|}
\hline Año & $\begin{array}{l}\text { Total Landfill } \\
\text { gas (m3/año) }\end{array}$ & $\begin{array}{l}\text { Energía térmica } \\
\text { (MWh/año) }\end{array}$ & $\begin{array}{c}\text { Energía } \\
\text { eléctrica } \\
\text { (MWh/año) }\end{array}$ & $\begin{array}{l}\text { Energía total de } \\
\text { cogeneración } \\
\text { (MWh/año) }\end{array}$ \\
\hline 2018 & 0,00 & 0 & 0 & 0 \\
\hline 2019 & $1.439 .742,99$ & 3.980 & 3.038 & 7.018 \\
\hline 2020 & $2.898 .135,81$ & 8.011 & 6.116 & 14.127 \\
\hline 2021 & $4.379 .754,14$ & 12.107 & 9.242 & 21.349 \\
\hline 2022 & $5.889 .289,06$ & 16.279 & 12.428 & 28.707 \\
\hline 2023 & $7.431 .562,34$ & 20.542 & 15.683 & 36.225 \\
\hline 2024 & $9.011 .542,25$ & 24.910 & 19.017 & 43.927 \\
\hline 2025 & $10.634 .359,96$ & 29.396 & 22.441 & 51.837 \\
\hline 2026 & $12.305 .326,64$ & 34.015 & 25.967 & 59.982 \\
\hline 2027 & $14.029 .951,17$ & 38.782 & 29.607 & 68.389 \\
\hline 2028 & $15.813 .958,79$ & 43.713 & 33.372 & 77.085 \\
\hline 2029 & $17.663 .310,37$ & 48.825 & 37.274 & 86.100 \\
\hline 2030 & $19.584 .222,81$ & 54.135 & 41.328 & 95.463 \\
\hline 2031 & $21.583 .190,22$ & 59.661 & 45.546 & 105.207 \\
\hline 2032 & $23.667 .006,25$ & 65.421 & 49.944 & 115.365 \\
\hline 2033 & $25.842 .787,54$ & 71.435 & 54.535 & 125.970 \\
\hline 2034 & $28.117 .998,33$ & 77.724 & 59.336 & 137.061 \\
\hline 2035 & $30.500 .476,38$ & 84.310 & 64.364 & 148.674 \\
\hline 2036 & $32.998 .460,32$ & 91.215 & 69.635 & 160.851 \\
\hline 2037 & $35.620 .618,38$ & 98.463 & 75.169 & 173.632 \\
\hline 2038 & $38.376 .078,81$ & 106.080 & 80.984 & 187.064 \\
\hline 2039 & $41.274 .461,88$ & 114.092 & 87.100 & 201.192 \\
\hline 2040 & $44.325 .913,79$ & 122.527 & 93.539 & 216.066 \\
\hline 2041 & $47.541 .142,40$ & 131.414 & 100.324 & 231.739 \\
\hline 2042 & $50.931 .455,05$ & 140.786 & 107.479 & 248.265 \\
\hline 2043 & $54.508 .798,58$ & 150.674 & 115.028 & 265.702 \\
\hline 2044 & $58.285 .801,66$ & 161.115 & 122.998 & 284.113 \\
\hline 2045 & $55.443 .169,57$ & 153.257 & 117.000 & 270.257 \\
\hline 2046 & $52.739 .174,28$ & 145.783 & 111.294 & 257.076 \\
\hline 2047 & $50.167 .054,4$ & 138.673 & 105.866 & 244.539 \\
\hline 2048 & $47.720 .378,28$ & 131.910 & 100.703 & 232.612 \\
\hline 2049 & 45.393.027,97 & 125.476 & 95.791 & 221.268 \\
\hline 2050 & $43.179 .183,88$ & 119.357 & 91.119 & 210.476 \\
\hline 2051 & $41.073 .310,23$ & 113.536 & 86.676 & 200.211 \\
\hline \multicolumn{5}{|c|}{ Continuación de la Tabla 6} \\
\hline 2052 & $39.070 .141,25$ & 107.999 & 82.448 & 190.447 \\
\hline 2053 & $37.164 .667,98$ & 102.731 & 78.427 & 181.159 \\
\hline 2054 & $35.352 .125,73$ & 97.721 & 74.602 & 172.323 \\
\hline 2055 & $33.627 .982,21$ & 92.955 & 70.964 & 163.919 \\
\hline 2056 & $31.987 .926,17$ & 88.422 & 67.503 & 155.925 \\
\hline 2057 & $30.427 .856,6$ & 84.109 & 64.211 & 148.320 \\
\hline 2058 & $28.943 .872,52$ & 80.007 & 61.079 & 141.087 \\
\hline TOTAL & $\begin{array}{c}\text { 1.226.945.217 } \\
00\end{array}$ & 3.391 .550 & 2.589 .178 & 5.980 .728 \\
\hline Promedio & $29.925 .493,10$ & 82.721 & 63.151 & 145.871 \\
\hline
\end{tabular}

En la figura 4 se observa la capacidad de generación de la central de cogeneración, obteniendo una capacidad máxima de generación de energía eléctrica de $122.998 \mathrm{MWh/año}$ y de generación térmica de 161.115 MWh/año en el 2044, luego de esta capacidad la generación de energía empieza a descender hasta llegar a una capacidad de generación de energía eléctrica y térmica de $61.079 \mathrm{MWh} /$ año y $80.007 \mathrm{MWh} / a$ ño respectivamente. Cabe notar que la generación de energía eléctrica y térmica está muy relacionada con la producción de biogás. 


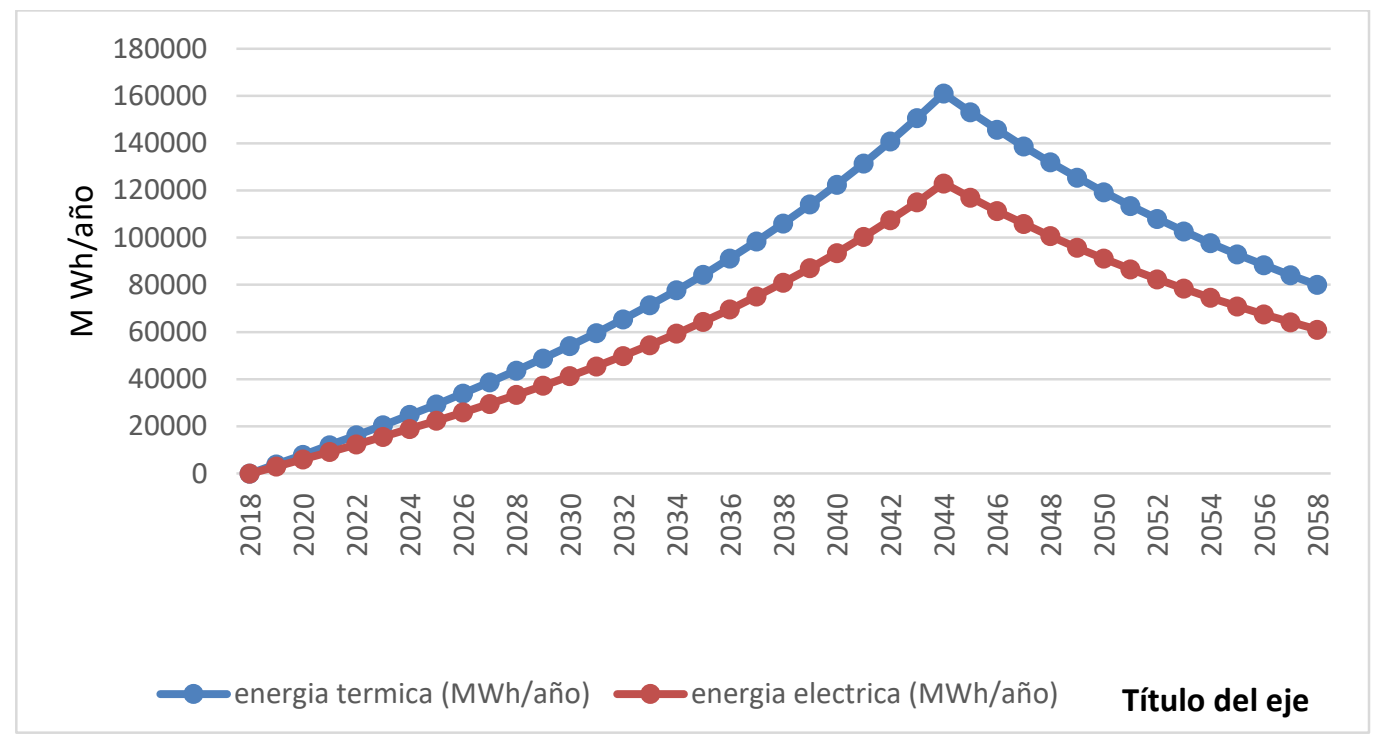

Figura 3. Energía cogenerada a partir de biogás y su variación temporal

Además, el total de la energía producida a partir del biogás del relleno sanitario de Juliaca desde el año 2018 hasta el año 2058 es de 5.980.728 MWh (3.391.550 MWh correspondientes a energía térmica y $2.589 .178 \mathrm{MWh}$ a energía eléctrica), con un promedio de $145871 \mathrm{MWh} /$ año de los cuales el promedio de energía térmica y eléctrica son 82.721 MWh/año y $63.151 \mathrm{MWh} /$ año respectivamente.

A partir de la información obtenida, se puede tener una visión general de la cantidad de biogás que puede producir el futuro relleno sanitario y el potencial que tiene para su aprovechamiento energético.

\section{CONCLUSIONES}

La estimación del biogás por medio del modelo matemático LandGEM resulto ser de mucha ayuda para poder calcular la cantidad de biogás que se puede producir en el relleno sanitario que se prevé se construirá en la ciudad de Juliaca.

Los resultados que arroja el programa comprende el periodo de 2018 hasta el año 2158, periodo en el cual se producirá biogás Según este modelo, y considerando una composición media del biogás de $50 \%$ de $\mathrm{CH}_{4}$ y $50 \%$ de $\mathrm{CO}_{2}$, y que comience la captura de biogás en el 2018 según los valores de $\mathrm{k}$ y $\mathrm{L}_{0}$ descritos, el potencial de recuperación de biogás estimado llegara a su punto más alto un año después del año de clausura, es decir, en el año 2044 con 58.285.801,66 m³ /año. Posteriormente disminuirá, año tras año. El promedio de biogás generado para los años de 2018 a 2058 corresponde a $29.925 .493,1 \mathrm{~m}^{3} /$ año.

La capacidad máxima de generación de la central de cogeneración, para la energía eléctrica es de $122.998 \mathrm{MWh} /$ año y de generación térmica de $161.115 \mathrm{MWh} / \mathrm{año}$ en el 2044, luego de esta capacidad la generación, la energía empieza a descender hasta llegar a una capacidad de generación de energía eléctrica y térmica de 61.079 MWh/año y 80.007 
MWh/año respectivamente. Además, el total de la energía producida a partir del biogás del relleno sanitario de Juliaca desde el año 2018 hasta el año 2058 es de 5.980.728 MWh (3.391.550 MWh correspondientes a energía térmica y 2.589.178 MWh a energía eléctrica), con un promedio de $145.871 \mathrm{MWh} /$ año de los cuales el promedio de energía térmica y eléctrica son $82.721 \mathrm{MWh} /$ año y $63.151 \mathrm{MWh} /$ año respectivamente.

\section{Referencias}

Aguilar, Q., Ojeda, S., Taboada, P., y Quintero, M. (2012). Estimación de las constantes k y Lo de la tasa de generación de biogás en sitios de disposicion final en baja California, México. Rev. Int. Contam. Ambie., 43-49.

Aguilar, Q., Taboada, P., y Ojeda, S. (2011). Potencial de producción eléctrica del biogás generado en un relleno sanitario. Ingenieria e Investigación, 56-65.

Aguirre, R., Camacho, I., y Solorzano, G. (s.f.). Metodologia para la determinacion de la emisión de gases invernadero de sitios de disposicion final de residuos sólidos municipales. ABES Asociasíon Brasíleña de Ingenieria Sanitaria y Ambiental.

Armenta-rivas, M., Sierra-Camargo, L., y Vélez-Pereira, A. (2017). Modelación de la producción de metano en el Relleno Sanitario Parque Ambiental Palangana. Ingenieria, Investigación y Tecnologia, 183-192.

Batool, S., y Chuadhry, M. (2008). el impacto de los metodos de tratamiento de los residuos sólidos municipales en las emisiónes de gases de invernadero en Lahore, Pakistan. Waste Management, 63-69.

Camargo, Y., y Vélez, A. (2009). Emisiónes de biogás producidas en rellenos sanitarios. REDISA.

Chamy, R., y Vivanco, E. (2007). Identificacion y clasíficacion de los distintos tipos de biomasa disponibles en Chile para la generación de biogás. Santiago de Chile: Proyecto de energías renovables no convencionales en Chile (CNE/GTZ).

Córdoba, V., Santalla, E., y Blanco, G. (2009). Modelado de la generación de biogás en rellenos sanitarios. Avances en Energías Renovables y Medio Ambiente.

IPCC. (2006). 2006 IPCC guidelines for National Greenhouse Gas Inventories, Prepared by the National Greenhouse Gas Inventories Programme. Japon: IGES.

Jaramillo, G., y Zapata, L. (2008). Aprovechamiento de los residuos sólidos orgánicos en Colombia. Antioquia: Universidad de Antioquia.

Machado, S., Carvalho, M., Gourc, J., Vilar, O., y Nascimento, J. (2009). Methane generation in tropical landfills: Simplified methods and field results. Waste Management, 153-161.

Martinéz, E., y Rodriguez, J. (2016). Aplicacion de las metodologias EPA, mexicano e IPCC para la estimación de biogás, caso de estudio relleno sanitario Doña Juana, Bogota. Universidad y Salud, 338-344.

Panesso, A., Cadena, J., Mora, J., y Ordoñez, M. (2011). Análisis del biogás captado en un relleno sanitario como combustible primario para la generación de energía eléctrica. Scientia et Technica, 23-28.

Pérez, M. (2006). producción de energa eléctrica a partir de biogás procedente de vertederos de residuos sólidos urbanos. Guatemala: Universidad de San Carlos.

Pignato, T., Aronica, S., Bonanno, A., Piazza, V., y Trapani, S. (2008). estimación del biogás producido en el vertedero de Palermo, aplicando un modelo Gaussian. Waste Management, 233-239.

Quintero, G., y Quintero, L. (2015). Perspectivas del potencial energético de la biomasa en el marco global y latinoamericano. Gestion y Ambiente, 179-188.

SCS Engineers. (2009). Manual de usuario Modelo Mexicano de Biogás Version 2.0. Obtenido de http://www.epa.gov/lmop/int/user_manual_mexico_lfg_model_v2_2009.pdf

Valladares, E., y Villegas, M. (2013). Potencial energético del biogás generado por los residuos Sólidos domiciliarios (RSD) en la provincia de Concepción. Concepción - Chile: Universidad del Bío - Bío. 